\title{
Prise en charge endovasculaire de l'AVC aigu
}

Kulcsar, Zsolt ; Carrera, Emmanuel ; Michel, Patrik

\begin{abstract}
The benefit of endovascular treatment (ET) after acute ischemic stroke has long been debated. Recent studies have demonstrated the clinical benefit of ET up to approximately 8 hours after stroke onset, mainly in association with intranenous thrombolysis. The positive effect is higher if ET is initiated early and performed by an experienced team. Current ET techniques include thrombectomy with clot removal using stentretriever technques or local thromboaspiration of the clot. ET seems to be so efficient that stroke care networks have to be organised to offer ET as quickly as possible in all patients who could benefit from this therapy.
\end{abstract}

DOI: https://doi.org/10.1056/NEJMoa1213701

Other titles: Endovascular treatment for acute ischemic stroke

Posted at the Zurich Open Repository and Archive, University of Zurich

ZORA URL: https://doi.org/10.5167/uzh-145395

Journal Article

Published Version

Originally published at:

Kulcsar, Zsolt; Carrera, Emmanuel; Michel, Patrik (2017). Prise en charge endovasculaire de l'AVC aigu. Revue Médicale Suisse, 13(560):886-889.

DOI: https://doi.org/10.1056/NEJMoa1213701 


\title{
Prise en charge endovasculaire de l'AVC aigu
}

\author{
Drs ZSOLT KULCSAR ${ }^{a}$, EMMANUEL CARRERA ${ }^{\mathrm{b}}$ et Pr PATRIK MICHEL
}

Rev Med Suisse 2017; 13: 886-9

\begin{abstract}
Le bénéfice du traitement endovasculaire (TEV) après AVC ischémique aigu est longtemps resté débattu. De nouvelles études ont démontré le bénéfice clinique du TEV jusqu'à 8 heures environ, en général, de préférence en combinaison avec un traitement intraveineux par thrombolyse au préalable. Cet effet à long terme a été rendu possible par le développement de nouvelles techniques endovasculaires comme le stent transitoire (stent retriever) permettant l'extraction du thrombus ou la thromboaspiration locale. Cette intervention est particulièrement efficace si elle est effectuée rapidement. Actuellement, des équipes expérimentées sont constituées dans des centres cérébrovasculaires pour offrir aux patients ce traitement dans les meilleurs délais.
\end{abstract}

\section{Endovascular treatment for acute ischemic stroke}

The benefit of endovascular treatment (ET) after acute ischemic stroke has long been debated. Recent studies have demonstrated the clinical benefit of ET up to approximately 8 hours after stroke onset, mainly in association with intranenous thrombolysis. The positive effect is higher if ET is initiated early and performed by an experienced team. Current ET techniques include thrombectomy with clot removal using stentretriever technques or local thromboaspiration of the clot. ET seems to be so efficient that stroke care networks have to be organised to offer ET as quickly as possible in all patients who could benefit from this therapy.

\section{INTRODUCTION}

Après des années d'incertitude au sujet des bénéfices des traitements endovasculaires (TEV) dans la phase aiguë de l'AVC ischémique, il existe maintenant des évidences scientifiques pour les recommander chez certains patients sélectionnés. Les bénéfices du TEV seront d'autant plus élevés si ce traitement est initié très rapidement après le début des symptômes dans un centre disposant d'une équipe expérimentée. Les techniques actuelles de TEV sont la thrombectomie par retrait du thrombus avec un stent transitoire (stent retriever) et l'aspiration de l'embole. Même si ces techniques s'adressent actuellement à une minorité de patients dans la phase aiguë de l'AVC, l'efficacité du TEV est telle que les systèmes de soins doivent tout mettre en œuvre pour offrir cette thérapie rapidement au plus grand nombre de patients potentiellement concernés. Nous décrivons ici les

a Service de neuroradiologie, ${ }^{\mathrm{b}}$ Centre cérébrovasculaire, Service de neurologie, Département des neurosciences cliniques, HUG, 1211 Genève 14, ${ }^{\mathrm{C}}$ Centre cérébrovasculaire, Service de neurologie, Département des neurosciences cliniques, CHUV, 1011 Lausanne patrik.michel@chuv.ch indications, la technique et les effets cliniques du TEV ainsi que l'organisation pré et intrahospitalière et les soins posttraitement endovasculaire.

\section{ORGANISATION PRÉHOSPITALIÈRE ET COMMUNICATION}

Au vu du bénéfice de la thrombolyse et des unités cérébrovasculaires (figure 1), il a été tout d'abord essentiel d'amener les patients souffrant d'AVC aussi vite que possible dans une unité cérébrovasculaire même avant l'utilisation de la thrombectomie. Les critères préhospitaliers pour identifier un candidat à la thrombolyse et/ou la thrombectomie sont très similaires:

- début aigu et très récent des symptômes (<5-7 heures);

- déficit neurologique handicapant;

- relative indépendance avant l'événement;

- absence de crise d'épilepsie au début des symptômes.

Environ un tiers des patients arrivant à l'hôpital dans les 6 premières heures ont une occlusion d'une artère cérébrale intracrânienne proximale et seraient donc candidats à une thrombectomie, en général dès le début de la thrombolyse intraveineuse. En réalité, l'éligibilité pour une thrombectomie pour tous les patients arrivant à l'hôpital dans les premières 6 heures est de l'ordre de $15-20 \%,{ }^{1}$ car en plus d'une occlusion artérielle intracrânienne, il faut une certaine indépendance fonctionnelle avant l'AVC, l'absence d'un volume majeur d'ischémie irréversible et un accès technique au vaisseau occlus. $^{2}$

Des patients avec une occlusion intracrânienne proximale peuvent être raisonnablement bien identifiés dans la phase préhospitalière, sur la base de critères cliniques, par plusieurs échelles (tableau 1). ${ }^{3-5}$ Des seuils relativement bas doivent

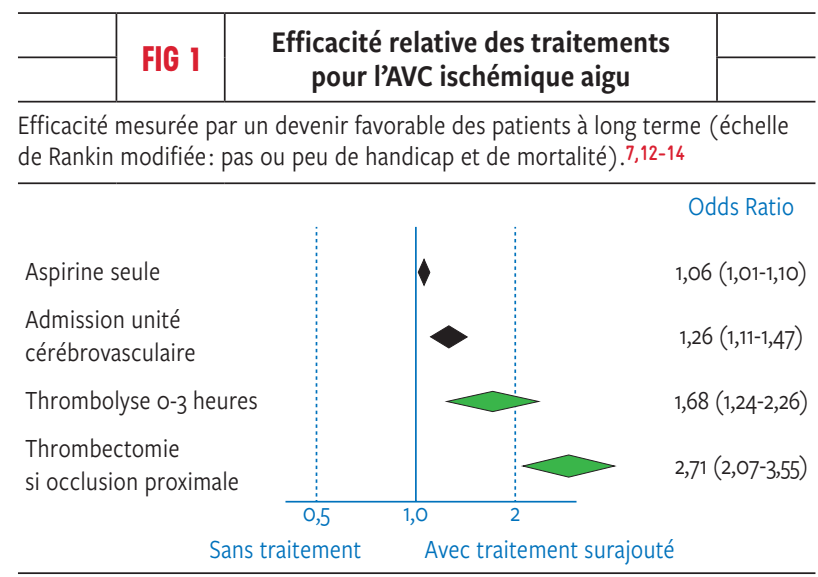




\section{\begin{tabular}{c|c|c|c}
\hline \multirow{2}{*}{ TABLEAU 1 } & $\begin{array}{c}\text { Identification préhospitalière des } \\
\text { patients susceptibles de bénéficier } \\
\text { d'une thrombectomie }^{3-5}\end{array}$ & \\
& &
\end{tabular}}

Echelles préhospitalières utilisées pour identifier les patients avec une probable occlusion artérielle intracrânienne majeure susceptibles de bénéficier d'une thrombectomie. Les cut-offs sont donnés pour assurer une sensibilité élevée, i.e. pour détecter $\geq 90 \%$ des occlusions artérielles proximales.

*en fonction des délais entre début et évaluation, ce seuil pourrait être plus haut.

\begin{tabular}{l|l|l}
\hline Scores & Noms complets & Cut-off \\
\hline G-FAST & Gaze-Face-Arm-Speech-Time & $\geq 3$ \\
\hline C-STAT (CPSSS) & $\begin{array}{l}\text { The Cincinnati Stroke Triage } \\
\text { Assessment Tool }\end{array}$ & $\geq 1$ \\
\hline LAMS & Los Angeles Motor Scale & $\geq 4$ \\
\hline PASS & Prehospital Acute Stroke Severity & $\geq 2$ \\
\hline RACE & Rapid Arterial Oclusion Evaluation & $\geq 2$ \\
\hline NIHSS & Full NIHSS & $\geq 7 *$ \\
\hline ASTRAL-O & ASTRAL-occlusion score & $\geq 13$ \\
\hline
\end{tabular}

être choisis avec ces échelles afin d'éviter d'exclure des patients potentiellement traitables. Cette contrainte mène automatiquement à un certain «surtriage» de patients admis dans un centre cérébrovasculaire (CCV) malgré l'absence d'une occlusion accessible au TEV. Alternativement, le modèle suisse des centres cérébrovasculaires (CCV; Stroke Centers) et des unités cérébrovasculaires (UCV; Stroke Units), effectuant une imagerie immédiate des artères cervico-cérébrales et initiant une thrombolyse ${ }^{6}$ permet à un patient proche d'une UCV d'être évalué et traité très rapidement. Seuls les patients qui remplissent les critères clinico-radiologiques dans l'UCV sont alors transférés sans délai au CCV. Une préannonce permet au centre receveur de préparer l'équipe et la salle d'angiographie où le patient arrivera directement depuis l'ambulance (ou l'hélicoptère).

\section{QUI BÉNÉFICIE D’UN TRAITEMENT \\ ENDOVASCULAIRE? \\ Identification clinique rapide des patients}

La méta-analyse de données individuelles des patients traités dans les grandes études randomisées montre un bénéfice similaire indépendamment de l'âge, du sexe, de la sévérité de l'AVC, et de la présence d'une occlusion ou d'une sténose simultanée d'une artère cervicale. ${ }^{7}$ De préférence, les patients ne devraient pas présenter un handicap significatif avant le traitement, et celui-ci est initié dans les 6 heures après la dernière preuve de bonne santé (tableau 2). ${ }^{2}$ De nouvelles analyses montrent par ailleurs un effet jusqu'à 7,3 heures, voire plus. ${ }^{8}$ Cependant, plus le délai entre symptômes et prise en charge devient important, plus l'analyse radiologique (collatérales, core (noyau ischémique), pénombre) devient importante dans la sélection des patients. Des études de thrombectomie jusqu'à 24 heures sont en cours, mais elles demanderont un volume d'ischémie irréversible encore plus petit qu'utilisé dans les premières 6-8 heures.

\section{Identification radiologique des patients}

L'intervalle de temps entre le début des symptômes et la recanalisation joue un rôle très important dans le succès clinique

\begin{tabular}{c|c|c|c}
\hline & \multirow{2}{*}{ TABLEAU 2} & $\begin{array}{c}\text { Indications certaines et relatives } \\
\text { pour la thrombectomi }\end{array}$ & \\
\cline { 1 - 1 } & & & \\
\end{tabular}

mesuré par l'échelle ASPECTS (Alberta Stroke Programe Early CT Score) ou avec la mesure du volume sanguin cérébral à l'imagerie de perfusion (ou de l'imagerie de diffusion à l'IRM). Mineur: qui devrait en général se situer $\geq 6$ points (ASPECTS) ou être diminué dans < 70-100 ml de tissu cérébral (IRM).

\begin{tabular}{|c|c|c|}
\hline Indications certaines & $\begin{array}{l}\text { Indications relatives, } \\
\text { avec évidence } \\
\text { scientifique limitée }\end{array}$ & $\begin{array}{l}\text { Facteurs sans } \\
\text { influence sur } \\
\text { l'efficacité }\end{array}$ \\
\hline $\begin{array}{l}\text { - Diagnostic d'AVC } \\
\text { ischémique aigu par un } \\
\text { spécialiste cérébrovasculaire, } \\
\text { après imagerie cérébrale (CT } \\
\text { ou IRM) } \\
\text { - Nouveau déficit neurologique } \\
\text { handicapant } \\
\text { - AVC traitable ( } \leq 6-8 \text { heures) } \\
\text { - Absence de handicap } \\
\text { significatif avant l'AVC } \\
\text { - Volume mineur d'ischémie } \\
\text { irréversible à l'imagerie* } \\
\text { - Occlusion d'artères } \\
\text { intracrâniennes proximales } \\
\text { (M1, siphon carotidien) }\end{array}$ & $\begin{array}{l}\text { - Amélioration rapide } \\
\text { des symptômes (avec } \\
\text { ou sans thrombolyse) } \\
\text { - Traitable entre } \\
\text { 8-24 heures si imagerie } \\
\text { favorable } \\
\text { - Occlusion d'artères } \\
\text { intracrâniennes distales } \\
\text { ou de la circulation } \\
\text { postérieure } \\
\text { - Handicape pré-AVC } \\
\text { modeste à modéré } \\
\text { - Volume majeur } \\
\text { d'ischémie irréversible* } \\
\text { - Absence de collatérales } \\
\text { ou de pénombre } \\
\text { significatives }\end{array}$ & $\begin{array}{l}\text { - Age } \\
\text { - Sexe } \\
\text { - Sévérité } \\
\text { de l'AVC } \\
\text { - Occlusion/ } \\
\text { sténose } \\
\text { simultanée } \\
\text { d'une artère } \\
\text { cervicale } \\
\text { - Patient ayant } \\
\text { reçu une } \\
\text { thrombolyse IV } \\
\text { ou pas }\end{array}$ \\
\hline
\end{tabular}

du traitement endovasculaire après AVC. Cependant, un autre facteur décisif pour la survie de tissu cérébral réside dans la capacité individuelle de suppléer le territoire en souffrance par la circulation collatérale. L'ischémie aiguë chez les patients sans réserve collatérale aboutira très rapidement à un infarctus définitif, même très tôt après la survenue de l'AVC. Ces sujets ne bénéficieraient pas, voire présenteraient une aggravation, si une revascularisation était réalisée pour reperfuser un territoire déjà infarci. Par opposition, d'autres patients, chez qui la circulation collatérale est capable de fournir un flux suffisant pour garantir la survie des zones de pénombre, bénéficieront plus longtemps des effets de la recanalisation. Dans ce contexte, le rôle principal de l'imagerie est d'identifier ces patients pour aider la prise de décision thérapeutique.

L'imagerie multimodale du tissu cérébral en situation d'urgence peut être réalisée par CT ou IRM. Les principales exigences de l'imagerie sont la capacité à identifier le core de l'infarctus, la vascularisation cérébrale et le site d'occlusion. D’importance secondaire, mais pouvant aider la prise de décision thérapeutique, est la visualisation de la pénombre (par imagerie de perfusion (CT de perfusion (CTP), IRM de perfusion (MRP)) et de la vascularisation collatérale. Le CT présente l'avantage d'être en général disponible 24 heures/24 et 7 jours $/ 7$, plus rapidement effectué, de meilleure qualité pour la visualisation des artères et du site d'occlusion, et de permettre un bilan précis de la circulation collatérale. Cependant, comparé à l'IRM, la sensibilité du CT pour la détection de l'infarctus dans la phase très précoce est moindre. Pour faciliter l'identification de la taille du core, le score ASPECT est également utilisé, permettant l'évaluation distincte de dix domaines différents du territoire de l'artère cérébrale moyenne (ACM). Une corrélation significative du score ASPECT initial avec le résultat clinique après revascularisation réussie a été prouvée.

L'imagerie par IRM a comme principal avantage la détection très précoce de l'infarctus grâce aux séquences pondérées de 
diffusion (DWI) avec une sensibilité très élevée. Dans la pratique quotidienne, la possibilité d'accéder à l'IRM en phase aiguë est en général moindre et demande une préparation plus longue des patients. De plus, cet examen est plus sensible aux artéfacts, notamment chez les patients non coopératifs. Il faut cependant noter que, par rapport au CT, le temps de l'examen n'est plus un facteur décisif, car l'information la plus importante peut être obtenue en quelques minutes avec des séquences IRM rapides.

L'une des raisons du succès des essais cliniques randomisés (RCT) a été la sélection des patients basée sur l'imagerie, en particulier le CT et la CT-angiographie (CTA). Il a été clairement prouvé que l'exigence minimale pour la sélection des patients pour la thrombectomie était, en plus de l'imagerie du parenchyme cérébral, l'imagerie des artères intracrâniennes, par CTA ou angiographie par IRM (MRA). L'imagerie par perfusion par CTP ou MRP permet également d'affiner la prise de décision.

Les trois questions les plus importantes à poser avant de prendre une décision pour le traitement endovasculaire sont les suivantes:

1. Le noyau (core) de l'infarctus est-il déjà trop grand? Il a été démontré que les volumes de core inférieurs à $70 \mathrm{ml}$ présentent le meilleur bénéfice après la revascularisation, tandis que ceux supérieurs à $100 \mathrm{ml}$ augmentent significativement le risque d'hémorragies de reperfusion. Une approximation de ces volumes est l'échelle ASPECTS (Alberta Stroke Programe Early CT Score) qui devrait en général se situer à $\geq 6$ points sur le CT natif ( $\geq 6$ points sur la DWI). Les patients dans la zone intermédiaire (taille du core 70-100 $\mathrm{ml}$ ) peuvent éventuellement encore bénéficier de la revascularisation. Dans ces cas spécifiques, la taille de la pénombre et les avantages potentiels guideront la décision.

2. Y at-il encore du tissu hypoperfusé qui peut être sauvé (pénombre?). L'imagerie de perfusion aidera à répondre selon les cartes CBV/MTT (figure 1). Lorsqu'aucun examen de perfusion n'est effectué, la taille de l'infarctus (core) doit être corrélée au score NIHSS. Une discordance (petit core et haut score NIHSS) parlera pour une pénombre persistante. La CTA (simple ou multiphase) peut fournir une approximation du réseau collatéral. Sur les séquences FLAIR de l'IRM, les signaux hyper-intenses dans les vaisseaux en aval de l'occlusion sont indicatifs d'un flux collatéral.

3. La troisième question est technique: $\mathrm{y}$ a-t-il une occlusion artérielle intracrânienne, et peut-on et avec quel risque accéder au site d'occlusion et effectuer une thrombectomie avec les outils endovasculaires actuellement disponibles? La majorité des patients atteints d'AVC sont âgés et présenteront des comorbidités cardiovasculaires, avec des vaisseaux aortique et supra-aortique tortueux et calcifiés, pouvant empêcher un accès facile.

Nos protocoles d'imagerie actuels sont plutôt basés sur le CT, avec des séquences natives, de perfusion et angiographiques (CTA). L'IRM sera réalisée dans des cas précis (incertitude sur le diagnostic, AVC constaté au réveil (wake-up stroke), occlusion basilaire avec suspicion d'infarctus du tronc cérébral étendu, par exemple). La sélection des patients basée sur l'imagerie est donc l'un des piliers les plus importants de la gestion réussie de l'AVC aigu. Ceci permet d'éviter des traitements futiles et

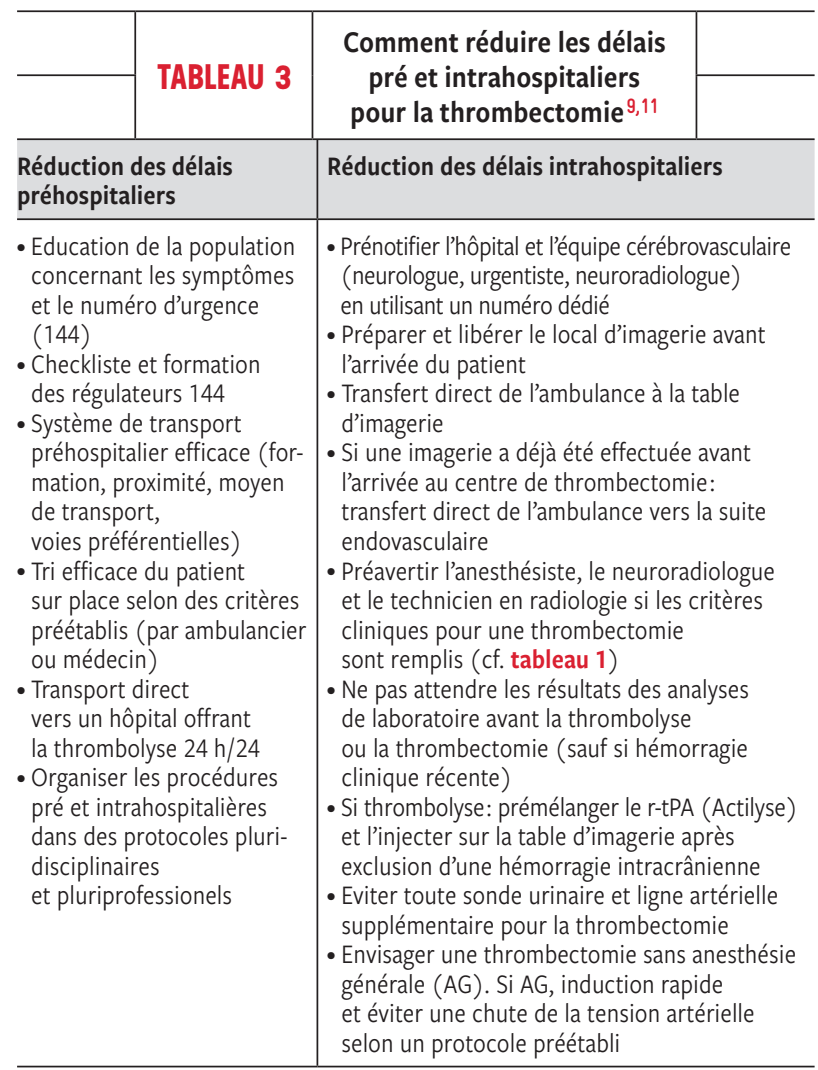

d'offrir la possibilité d'une revascularisation endovasculaire à tous les patients qui pourraient en bénéficier.

\section{RÉDUCTION DES DÉLAIS PRÉ ET INTRAHOSPITALIERS}

Le temps étant primordial pour le succès de la thrombolyse et de la thrombectomie, des efforts majeurs sont entrepris pour raccourcir les délais pré et intrahospitaliers. Le tableau 3 liste de telles mesures dont la majorité a maintenant une base scientifique. ${ }^{9}$ En mesurant constamment les délais et en fournissant un feedback aux intervenants, ces délais sont constamment réduits. Aussi, des exercices de simulation sont maintenant utilisés dans l'entraînement des équipes impliquées.

\section{THROMBECTOMIE: TECHNIQUE ACTUELLE}

L'objectif du traitement endovasculaire est d'éliminer en une seule pièce le thrombus occlusif de l'artère cérébrale dans laquelle il se trouve sans endommager le système vasculaire et sans fragmenter le caillot. Ceci peut provoquer une embolisation distale ou dans un nouveau territoire vasculaire, a priori non affecté. Le «breakthrough» du traitement endovasculaire a été atteint par les «stent retrievers», qui sont des stents cérébrovasculaires, auto-expansibles, attachés à un fil de livraison. Le dispositif est déployé à travers un microcathéter au niveau de l'occlusion. En s'ouvrant, par expansion radiale le long de l'axe longitudinal du dispositif, la structure du stent pénètre dans le caillot. Dans cette position ouverte, le stent est ensuite retiré, entraînant également le thrombus attaché. L'efficacité de cette technique a été évaluée dans les RCT et a montré un bénéfice significatif de la thrombectomie mécanique 
par rapport au traitement par lyse intraveineuse. Cet effet augmentait avec la taille du vaisseau occlus. Le degré de la revascularisation est mesuré avec les score TICI (Treatment In Cerebral Ischemia Scale), les scores TICI $2 b$ et 3 correspondant à un succès radiologique. Les études randomisées ont démontré qu'une désoblitération efficace était une des clés d'un bon résultat clinique.

Un autre traitement de désobstruction encore en développement est la thromboaspiration, qui connaît aujourd'hui un deuxième souffle. Les progrès technologiques axés sur les besoins cliniques ont donné lieu à la mise au point de cathéters à lumière large (jusqu'à $6 \mathrm{~F}$ ), suffisamment flexibles pour être guidés dans le système vasculaire intracrânien jusqu'au contact avec le thrombus, sans traumatiser les vaisseaux cérébraux. A travers la grande lumière du cathéter, une force d'aspiration plus élevée peut être exercée, soit par une pompe d'aspiration, soit à l'aide d'une seringue. Ceci conduira à l'élimination du thrombus, soit par ingestion, soit de par sa capture par le cathéter, qui sera ensuite retiré. L'inconvénient de la thromboaspiration est le risque de fragmentation du thrombus. Les avantages sont la rapidité de la procédure et l'effet traumatique moins important sur la paroi du vaisseau. En cas de défaillance, il peut être facilement combiné avec la technique du stent retriever. L'efficacité de cette procédure n'a pas encore été démontrée, l'étude randomisée ayant été arrêtée prématurément en raison de la positivité des études avec stent retrievers. ${ }^{10}$

La question des bénéfices et risques d'une éventuelle anesthésie générale (AG) pour la thrombectomie (vs sédation consciente) reste actuellement ouverte. Les avantages de l'AG sont le confort du patient pour une procédure souvent douloureuse, et l'évitement de mouvements et de l'agitation pendant l'intervention. Les désavantages sont le risque de chute de la tension artérielle ainsi que les complications d'une intubation prolongée, si l'extubation ne peut pas se faire rapidement après l'AG.

\section{APRÈS LA THROMBECTOMIE: IL EST IMPORTANT DE POURSUIVRE LES SOINS D’EXCELLENCE}

Si une thrombolyse a précédé la thrombectomie, les antithrombotiques (en général charge d'aspirine 250-500 mg) ne peuvent être donnés qu'après que l'absence d'une hémorragie importante ait été confirmée par l'imagerie 12-24 heures après la thrombolyse. Après une thrombectomie pure, cette dose de charge peut être donnée immédiatement à la fin de la procédure. Un traitement par statine est continué (ou initié) aussi rapidement que possible, et la tension artérielle (<185/105 pendant la procédure) est maintenue à des valeurs légèrement élevées lors d'une recanalisation complète par thrombectomie.

\section{CONCLUSION}

L'admission dans une UCV ou un CCV est un facteur de bon pronostic (figure 1) qui devrait être offerte à tous les patients cérébrovasculaires. Dans ces unités, une mobilisation rapide sur 12-24 heures (si toléré) est favorisée, et la rééducation commence le deuxième jour après l'AVC selon des critères bien établis. ${ }^{6}$ Le bilan étiologique et des facteurs de risque est complété, et des évaluations neuropsychologiques et de neurorééducation sont effectuées. La prévention secondaire précoce est renforcée et les patients et proches bénéficient souvent de séances d'éducation thérapeutique par du personnel médical spécialement formé.

Conflit d'intérêts: Le Pr Patrik Michel a reçu du soutien pour la recherche et formation de Boehringer-Ingelheim et Medtronic.

Les autres auteurs n'ont déclaré aucun conflit d'intérêts en relation avec cet article.

\section{IMPLICATIONS PRATIOUES}

- Un système de triage préhospitalier et de traitement bien structuré est indispensable au traitement rapide par thrombolyse et thrombectomie (time is brain)

- Le traitement endovasculaire aigu est devenu un traitement hautement efficace chez les patients sélectionnés par imagerie dans les unités et centres cérébrovasculaires

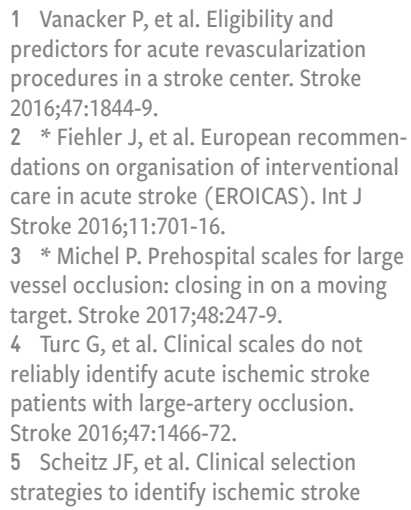

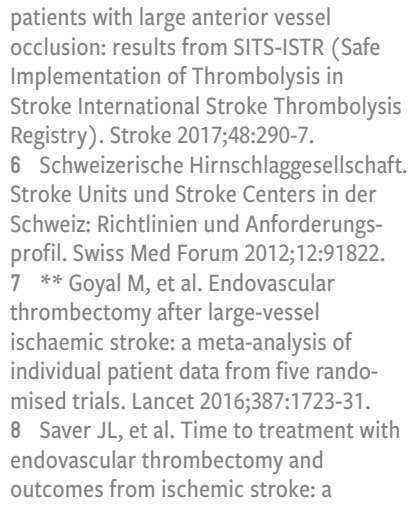

patients with large anterior vessel occlusion: results from SITS-ISTR (Safe Implementation of Thrombolysis in Stroke International Stroke Thrombolysis Registry). Stroke 2017;48:290-7. 6 Schweizerische Hirnschlaggesellschaft. Stroke Units und Stroke Centers in der Schweiz: Richtlinien und Anforderungsprofil. Swiss Med Forum 2012;12:91822. $7 * *$ Goyal M, et al. Endovascular thrombectomy after large-vessel ischaemic stroke: a meta-analysis of individual patient data from five randomised trials. Lancet 2016;387:1723-31.

8 Saver JL, et al. Time to treatment with endovascular thrombectomy and

outcomes from ischemic stroke:

meta-analysis. JAMA 2016;316:1279-88. $9 * *$ Xian Y, et al. Use of strategies to improve door-to-needle times with tissue-type plasminogen activator in acute ischemic stroke in clinical practice: findings from target: stroke. Circ Cardiovasc Qual Outcomes 2017;10. 10 Mocco J, et al. Aspiration thrombectomy after intravenous alteplase versus intravenous alteplase alone. Stroke 2016;47:2331-8.

11 Meretoja A, et al. Reducing in-hospital delay to 20 minutes in stroke thromboly sis. Neurology 2012;79:306-13.

12 Langhorne $\mathrm{P}$, on behalf of the Stroke Unit Trialists' Collaboration. Organized inpatient (Stroke Unit) care for stroke.
Antiplatelet therapy for acute ischaemic stroke. Cochrane Review. Cochrane Library 2005;1-36.

13 Langhorne $P$., on behalf of the Stroke Unit Trialists' Collaboration. Organized inpatient (Stroke Unit) care for stroke. Stroke 2014;45:e14-5.

14 Emberson J, et al. Effect of treatment delay, age, and stroke severity on the effects of intravenous thrombolysis with alteplase for acute ischaemic stroke: a meta-analysis of individual patient data from randomised trials. Lancet 2014;384:1929-35.

* à lire

** à lire absolument 